\title{
Nonlinear dynamics of the patient's response to drug effect during general anesthesia
}

\author{
Clara Ionescu, Jose Tenreiro Machado, Robin De Keyser, Johan Decruyenaere, \\ Michel M.R.F. Struys
}

A B S T R A C T

In today's healthcare paradigm, optimal sedation during anesthesia plays an important role both in patient welfare and in the socio-economic context. For the closed-loop control of general anesthesia, two drugs have proven to have stable, rapid onset times: propofol and remifentanil. These drugs are related to their effect in the bispectral index, a measure of EEG signal. In this paper wavelet time-frequency analysis is used to extract useful infor-mation from the clinical signals, since they are time-varying and mark important changes in patient's response to drug dose. Model based predictive control algorithms are employed to regulate the depth of sedation by manipulating these two drugs. The results of identifi-cation from real data and the simulation of the closed loop control performance suggest that the proposed approach can bring an improvement of $9 \%$ in overall robustness and may be suitable for clinical practice.

\section{Keywords:}

Bispectral index

Depth of anesthesia

Drug delivery

Identification

Predictive control

Wavelet analysis

\section{Introduction}

Today's socio-economic context puts a lot of pressure on the standard practices and guidelines in intensive care unit and operation theater. Stringent regulations concerning patient safety and welfare have motivated anesthesiologists to allow more and more the use of closed loop control within the clinical practice [1]. From the latest advances in closed loop control of depth of anesthesia, it became clear that a patient-individualized model for drug effect predictions is crucial in ensuring optimal performance within the imposed constraints [2].

The earliest controllers developed for general anesthesia are of the Proportional-Integrative-Derivative (PID) type [3]. Due to the fact that PID controllers cannot anticipate to the response of the patient and have limited robustness, stability problems may arise. Therefore, strategies using model based predictive control (MPC) [4] and Bayesian-based control algorithms $[5,6]$ have been suggested and successfully applied in practice. The current bottleneck is the specific characterization of the multiple drug input and its synergistic effect upon the depth of anesthesia for personalized healthcare. 
In general, biological signals and systems are non-stationary and contain time delays [7,8]. The efficiency of wavelet tools employed to extract useful information for anesthesia has been shown in several recent works [9]. In this paper, we employ clinically available signals from intensive care unit clinical trials executed at the Ghent University Hospital, Belgium. The signals are recorded from patients under general anesthesia during their stay at the intensive care unit and are the result of manual control, i.e. of a specialized clinical nurse who is keeping the patient's vital signs stable.

It has been shown that these two drugs, i.e. propofol and remifentanil, have synergic effects on hypnosis level and can be administrated together for a suitable depth of anesthesia regulation. The pharmacodynamic relation between the drug concentrations in the body and their effect can be characterized by a nonlinear surface known as the Sigmoid Hill curve. Since this surface is nonlinear with respect to the variations in the drug concentrations in blood, it is difficult to perform identification and the global solution is not always feasible due to safety constraints. During their stay at the intensive care unit, the anesthestized patient is maintained within a specific interval of the electrical activity in the brain, measured by electroencephalogram (EEG). Since the EEG is rather noisy and complex, several data channels are combined by means of signal processing techniques and a singular value is delivered, between $0-100 \%$, i.e. isoline EEG and fully awake patient respectively. This singular value is known as the dimensionless Bispectral Index (BIS) and sensors are commercially available for the clinical practice. The exact details of the algorithm used to create the BIS index have not been disclosed by the company that developed it (Aspect Medical Systems). By having this measure at hand, a linear model can be approximation, since the nonlinear surface is piecewise linear. The results of such a linear identification procedure are presented in this work, along with an evaluation of closed loop control. Several simulations are performed, which demonstrate that the inter-patient variability is tackled successfully by means of this personalized closed loop predictive control algorithm.

Having these ideas in mind, the paper is structured as follows. Section 2 presents the clinical data used for identification, the modeling approach to describe drug interaction, the signals filtering and the MPC strategy. Section 3 develops the identification and the control algorithms. The results obtained are discussed in detail in Section 4. A conclusion section summarizes the main outcome of this paper.

\section{Materials and methods}

\subsection{Clinical data}

The data used for the identification purpose in this paper is a selection of a larger study ongoing at Ghent University Hospital, where clinical trials have been performed during the period September 2007-May 2010. Written informed consent was obtained from the patient or legal representative. The study protocol was approved by the local Ethics Committee of Ghent University Hospital, and was performed in accordance with the Declaration of Helsinki and the Good Clinical Practice Guideline of the European Commission. Patients were routinely regarded eligible for inclusion according to the following criteria: i) patients in the immediate post-operative phase after a coronary artery bypass graft (CABG) surgery and ii) age higher than 18 years.

Data from 5 patients receiving manually controlled propofol and remifentanil sedation was selected for this study. An existing and previously validated software platform was used to record the data and to control the syringe pumps delivering the drugs to these patients, i.e. RUGLOOP II, Demed, Temse, BELGIUM [10]. Target-controlled infusion technology available in RUGLOOP II was applied during drug delivery. Measurements were performed every second, using the standard Aspect Medical Brain Monitoring System, which delivers the BIS signal values to a clinical interface. The BIS was acquired using standard 3-4 electrode sensors, adjustable on adult head frontal The biometric values of the patients selected for this study are given in Table 1.

The clinical trials have been performed during $6 \mathrm{~h}$. In most of the patients, after the first hour, the peri-operative drugs are not active anymore and the patient is less deeply sedated.

\subsection{Patient model}

The most commonly used drugs for anesthesia in Belgium are propofol and remifentanil. They have a reliable pharmacological profile, with fast onset and recovery times, making them suitable candidates for closed loop control of depth of anesthesia.

Table 1

Biometric values of the patients selected for this study; BMI: body mass index.

\begin{tabular}{llllll}
\hline Patient & Age (years) & Length $(\mathrm{cm})$ & Weight $(\mathrm{kg})$ & Gender & BMI \\
\hline 1 & 53 & 186 & 114 & $\mathrm{M}$ & 32 \\
2 & 71 & 172 & 83 & $\mathrm{M}$ & $\mathrm{M}$ \\
3 & 72 & 162 & 87 & $\mathrm{M}$ & 33 \\
4 & 61 & 182 & 93 & $\mathrm{M}$ & 28 \\
5 & 69 & 158 & 81 & - & 32 \\
Mean \pm Std & $62.5 \pm 9.5$ & $172 \pm 14$ & $97.5 \pm 16.5$ & & $30.6 \pm 2.4$ \\
\hline
\end{tabular}


Propofol is a hypnotic agent, whose pharmacological properties have been well described and studied in different kind of patients [11,12]. Remifentanil is an opioid with a unique pharmacological profile, best characterized by its high metabolic clearance, independent of the most common metabolic pathways which are usually known to metabolize anesthetic drugs $[13,14]$. When administered together, these two drugs interact synergistically on both hypnotic and analgesic components of sedation. Their combined effect is characterized by the BIS, a signal derived from the electroencephalogram (EEG) [15]. Using EEG, several derived, computerised parameters like the BIS have been tested and validated as a promising measure of the hypnotic component of anesthesia [21]. BIS combines several features extracted from EEG including higher order spectra of the signal which can reveal phase coupling of single waveforms. Multivariate statistics were used to combine the different features into a single indicator value [22]. BIS values are given in units of percentage of hypnosis and lie in the range of $0-100 \%$; whereas $90-100 \%$ range represents fully awake patients, $60-70 \%$ range indicate light hypnosis and $40-60 \%$ range indicate moderate hypnotic state. For patient safety and fast recovery time, BIS should not decrease during clinical interventions below $30 \%$ and should not increase above $70 \%$.

Some artifacts may be present in the measured BIS signal, e.g. due to body movement, eye movements, coughing, sweating, etc. A typical BIS signal with body movement and coughing artefacts is presented in Fig. 1. The task of the anesthesiologist is to prevent those high amplitude values by injecting the appropriate drug dose. This is a complex task, due to the intra- and inter-patient variability and automatic control of general anesthesia is a challenging demand.

Pharmaco-kinetic and pharmaco-dynamic (PK-PD) blocks denote compartmental models. The PK-PD models most commonly used for propofol and remifentanil are the 4th order compartmental model depicted in Fig. 2.

The compartmental model can be represented by a set of equations:

$$
\left[\begin{array}{c}
\dot{x_{1}} \\
\dot{x_{2}} \\
\dot{x_{3}} \\
\dot{C_{e}}
\end{array}\right]=\left[\begin{array}{cccc}
-k_{10}+k_{12}+k_{13} & k_{21} & k_{31} & 0 \\
k_{12} & -k_{21} & 0 & 0 \\
k_{13} & 0 & -k_{31} & 0 \\
k_{1 e} & 0 & 0 & -k_{e 0}
\end{array}\right] \cdot\left[\begin{array}{l}
x_{1} \\
x_{2} \\
x_{3} \\
C_{e}
\end{array}\right]+\left[\begin{array}{l}
1 \\
0 \\
0 \\
0
\end{array}\right] \cdot u .
$$

where $x_{1}[\mathrm{mg}]$ denotes the amount of drug in the central compartment. The blood concentration can be expressed by $x_{1} / V_{1}$, where $V_{1}$ is the volume of blood in the central compartment. The peripheral compartments 2 and 3 model the drug exchange of the blood with well (i.e. muscles) and poorly (i.e. fat) diffused body tissues. The masses of drug in fast and slow equilibrating peripheral compartments are denoted by $x_{2}$ and $x_{3}$, respectively. The parameters $k_{j i}$, for $i \neq j$, denote the drug transfer frequency from the $j$ th to the $i$ th compartment and $u(t)[\mathrm{mg} / \mathrm{s}]$ is the infusion rate of the drug into the central compartment.

The additional hypothetical effect compartment represents the lag between drug plasma concentration and drug response. The amount of drug in this compartment is represented by $C_{e}$. The parameters $k_{i j}$ of the PK models depend on age, weight, height and gender and the relations can be found for propofol in $[11,12]$ and for remifentanil in $[13,14]$. This hypothetical effect compartment makes the link to the drug effect, i.e. BIS values, by a sigmoid nonlinear, time-varying curve as represented in Fig. 3. This is also the nonlinear drug/effect relation block from Fig. 2. The typical sigmoid shape relating the BIS values to the effect site concentration $C_{e}$, starts with a flat plateau. During this time no closed loop feedback control can be applied since there is no useful feedback signal available the Propofol drug is infused into the patient while BIS values do not vary significantly; moreover, in practice the little variation is hidden in noise. In practice, during this time, manual control is applied until BIS values show a certain variation $\Delta \mathrm{BIS}$. If the desired BIS value is $\mathrm{BIS}=50 \%$, then the concentration

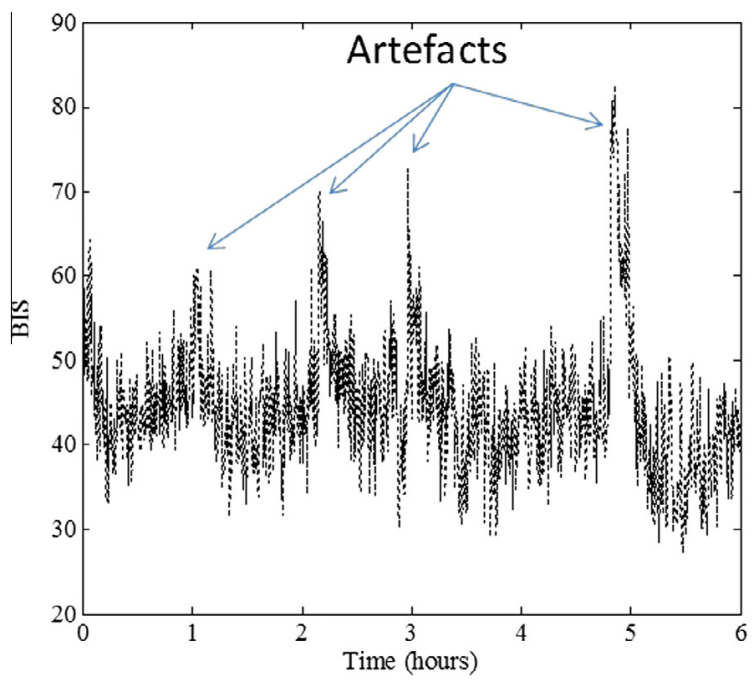

Fig. 1. Real BIS signal where artifacts can be observed. First artefact is coughing and the other three are due to body movement. 


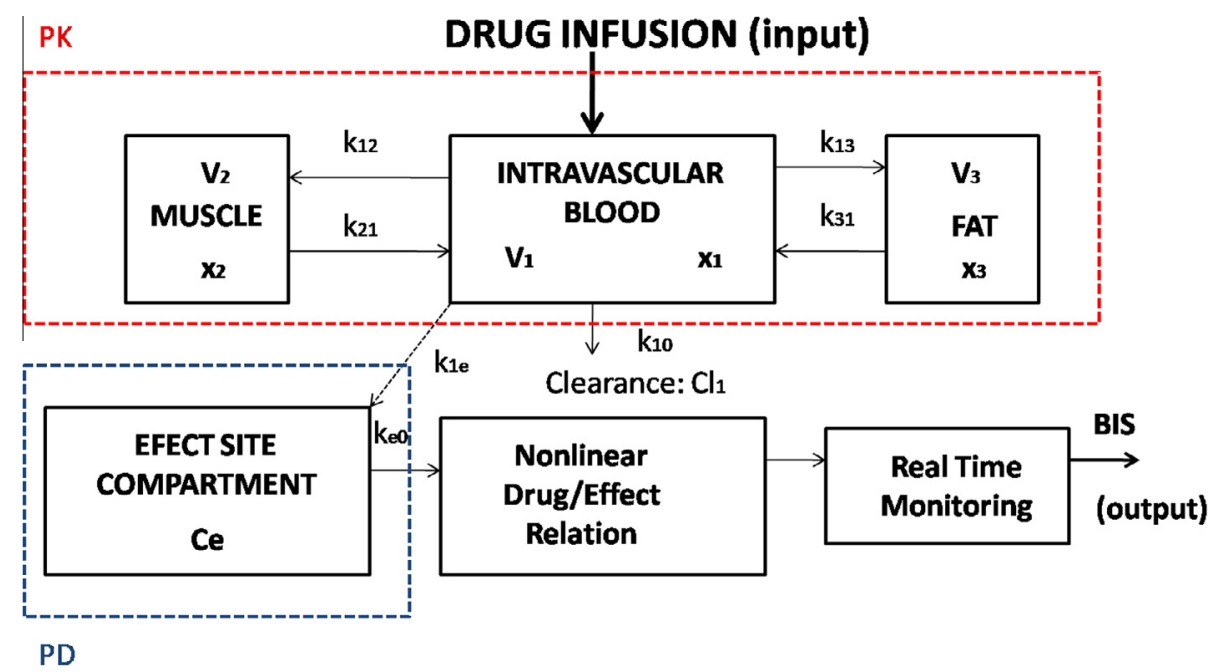

Fig. 2. General compartmental model of the patient, where PK denotes the pharmacokinetic model and PD denotes the pharmacodynamic model of an infused drug.

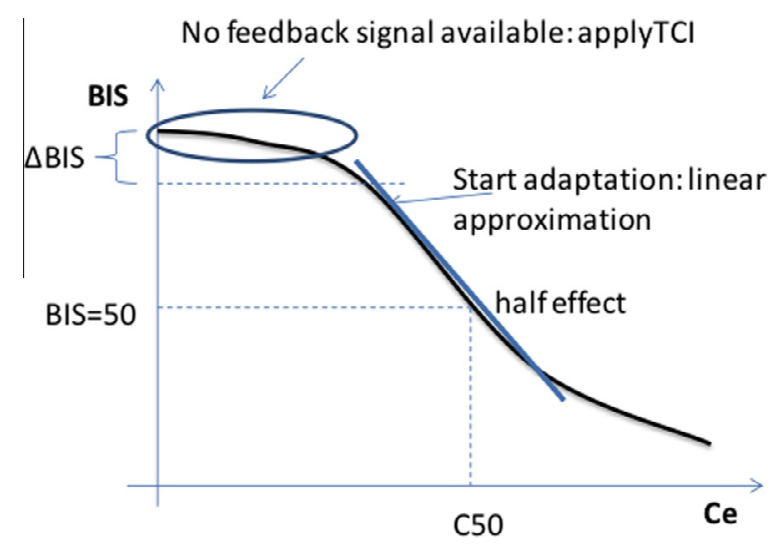

Fig. 3. Sigmoid curve of the effect from one drug. TCI stands for target controlled infusion, the standard practice in clinical trials (i.e. open loop control).

needed to be achieved in the blood of the patient is denoted by $C_{50}$. This values is usually some averaged value over a large population database and can be adopted as a starting point of adaptation of the patient specific model.

When considering the effect of two drugs, the nonlinear drug/effect relation, represented by the block from Fig. 2 is in fact a 3D function, leading to a nonlinear surface representing the synergistic effect of both propofol and remifentanil drugs.

The nonlinear complex synergy of the two drugs can be described by a normalized, time-varying relation [15]:

$$
B I S(t)=E_{0}-E_{\max }(\theta) \cdot \frac{\left(\frac{U_{\text {Prop }}(t)+U_{\text {Rem }}(t)}{U_{50}(\theta)}\right)^{\gamma(\theta)}}{1+\left(\frac{U_{\text {Prop }}(t)+U_{R e m}(t)}{U_{50}(\theta)}\right)^{\gamma(\theta)}},
$$

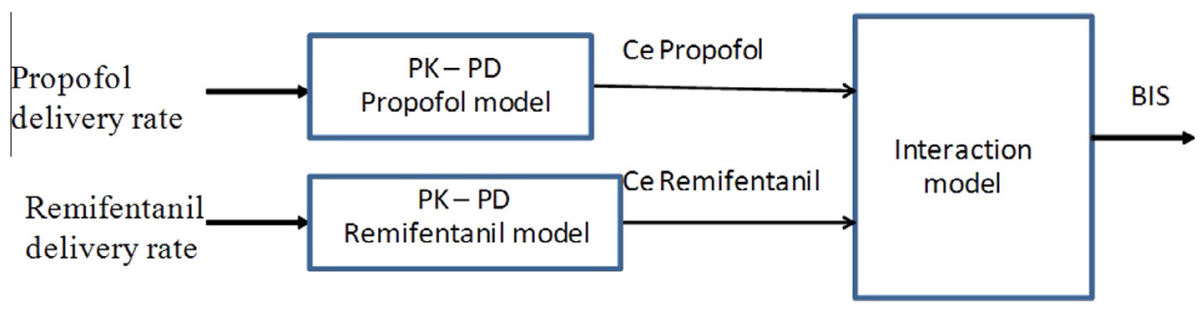

Fig. 4. Block diagram of the patient model for two drug inputs and one output effect. 


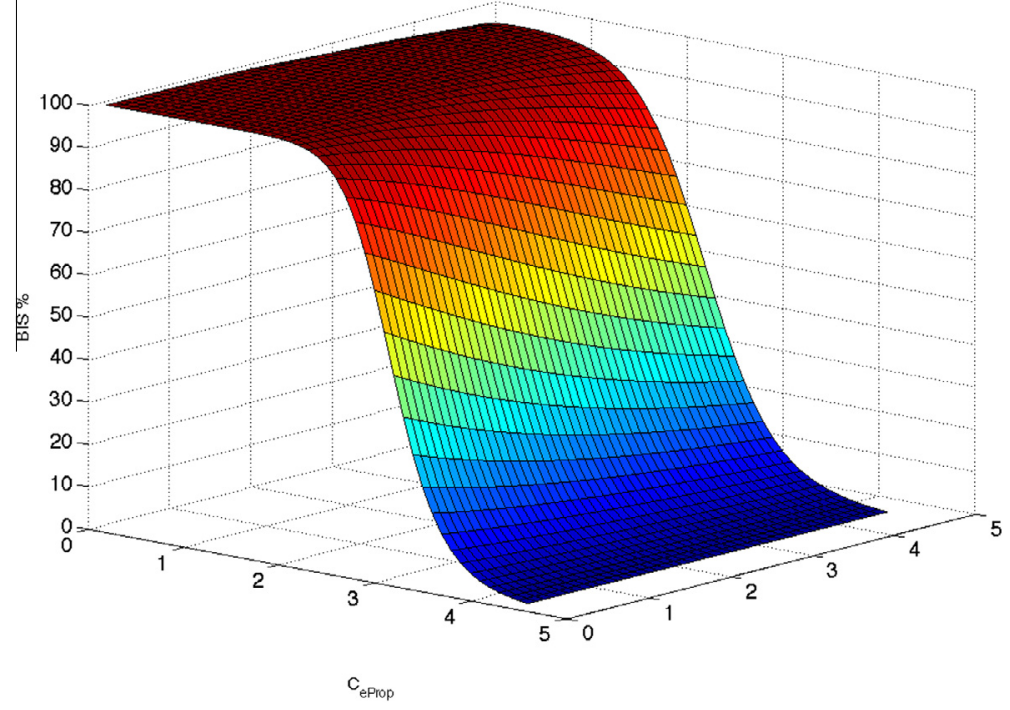

Fig. 5. Sigmoid surface of the effect from two drugs.

where: $U_{\text {Prop }}(t)+U_{R e m}(t)$ is the combined drug concentration; $\gamma(\theta)$ is the steepness of the concentration-response relation at ratio $\theta ; U_{50}(\theta)$ is the number of drug units associated with $50 \%$ of maximum effect at ratio $\theta ; E_{\max }(\theta)$ is the maximum possible drug effect at ratio $\theta$. The effect-site concentrations $C_{e \text { Prop }}$ and $C_{e \text { Rem }}$ are normalized to their respective potencies $C_{50 \text { Prop }}$ and $C_{50 \text { Rem }}$ as described by:

$$
U_{\text {Prop }}(t)=\frac{C_{e \text { Prop }}(t)}{C_{50 \text { Prop }}} ; U_{\text {Rem }}(t)=\frac{C_{e R e m}(t)}{C_{50 R e m}} ;
$$

and the time-varying ratio of the interacting drugs is expressed by:

$$
\theta(t)=\frac{U_{\text {Prop }}(t)}{U_{\text {Prop }}+U_{\text {Rem }}} ;
$$

In this formulation, $\theta$ represents the concentration ratio of the new combined drug and ranges from 0 (Remifentanil only) to 1 (Propofol only). The values for $E_{\max }(\theta)$ and $E_{0}$ are usually set to $100 \%$ and $U_{50}(\theta)$ can be expressed by a quadratic polynomial:

$$
U_{50}(\theta)=1-\beta \cdot \theta+\beta \cdot \theta^{2} .
$$

The unknown parameter $\beta$ is usually estimated from the patient data. Since the interaction between the two drugs is supraadditive (the effect of the two drugs combined is higher than the sum of each separate effect), then $\beta>0$. This means that $U_{50}(\theta)<1$ for $0<\theta<1$. However, in the application presented in this paper, this model is applied to patients in intensive care unit, i.e. BIS should be maintained within an interval of [40-60\%]. Consequently, the highly nonlinear surface from (2) can be approximated just by a linear function:

$$
B I S(t)=m_{1} \cdot C_{e P r o p}\left(t-\tau_{d}\right)+m_{2} \cdot C_{e R e m}\left(t-\tau_{d}\right)+b,
$$

where $m_{1}$ and $m_{2}$ are the slopes, $b$ is a constant and $\tau_{d}$ is the time delay introduced by the signal processing of the BIS monitor [16].

\subsection{Wavelet transformation and identification}

During general anesthesia, the patient's response to the infused drug is varying considerably. This results in non-stationary signals which change their power spectral density function in time. Since all models used for prediction of drug infusion rates are based on a general population dataset, it is obvious that they will no longer correspond to the actual dynamics of each particular patient. Hence, it is necessary to obtain suitable models that can be easily adapted online to the intra-patient variability and avoid over- and under-sedation.

In order to analyze a non-stationary signal in time-frequency domain, wavelet analysis can be employed, by multiplying the non-stationary signal $x(t)$ by a function [17]. Considering the mother wavelet function $\psi$ centered at time instant $\tau$ and scaled by a factor $s$, the continuous wavelet transform (CWT) of the signal $x(t)$ is represented by [18]:

$$
C W T(s, \tau)=\frac{1}{\sqrt{|S|}} \int_{-\infty}^{+\infty} x(t) \cdot \psi\left(\frac{t-\tau}{s}\right) d t .
$$


The digital version of the CWT is the discrete wavelet transform (DWT). A way to implement the DWT using filters was developed by Mallat [18]. Filters of different cut-off frequencies are used to separate the signal. The concept is schematically illustrated in Fig. 6, where $g_{i}$ denote low-pass filtering and $h_{i}$ denote high-pass filtering. Specific details are given in the 'Results' section.

At the first level, the signal is decomposed simultaneously using a low pass and high pass filter. This procedure is repeated and the approximation coefficients are decomposed again with high and low pass filters. There are several wavelet families proposed by different researchers. The wavelet used in this study is the Haar function, due to its small computational time, represented by the following formula:

$$
\psi(t)= \begin{cases}1 & 0 \leqslant t<\frac{1}{2} \\ -1 & \frac{1}{2} \leqslant t<1 \\ 0 & \text { otherwise }\end{cases}
$$

For the application in this study, we were interested to capture the intra-patient variability from the filtered BIS signal. Therefore, the total length of the BIS signal was divided in several windows and identification applied on each data interval, as detailed in the 'Results' section. The unknown parameters of the linear approximation (6), namely $m_{1}, m_{2}$ and $b$ were identified using the classical least-squares method. The time-delay $\tau_{d}$ was estimated based on cross-correlation analysis [16].

\subsection{MPC-EPSAC strategy}

The inherent capability of MPC to outperform other control strategies has been already shown in [19,4] by means of simulations evaluating both the performance and the robustness of the closed loop system. In the general MPC scheme represented in Fig. 7, the patient model is used to predict the future value of the output variable BîS. The difference between the measured BIS from the patient and the model output (residual), serves as feedback signal in the prediction block. With this residual and the input $u$, the prediction block predicts the future values of the output BIS. On the basis of these predicted BIS values, the controller calculates the future optimal drug infusion rates over a number of samples in the future, called the prediction horizon and denoted by $N_{2}$ samples. However, only the first calculated sample value is applied to the process (i.e. principle of receding horizon). Notice that this algorithm is naturally based on digital control (sampled data).

In this paper, the EPSAC (Extended Prediction Self-Adaptive Control) strategy is employed [20]. The EPSAC algorithm is based on a generic process model:

$$
B I S(t)=B \hat{I} S(t)+n(t),
$$

with $t$ denoting time instants. The term $n(t)$ includes the effects in the measured output $B I S(t)$ which do not come from the model input $u(t)$ via the available model output $B I S(t)$. The information carried by this term is a combination of twofold origins: i) modeling errors and ii) physical disturbances on the measured signal coming from the measurement equipment,

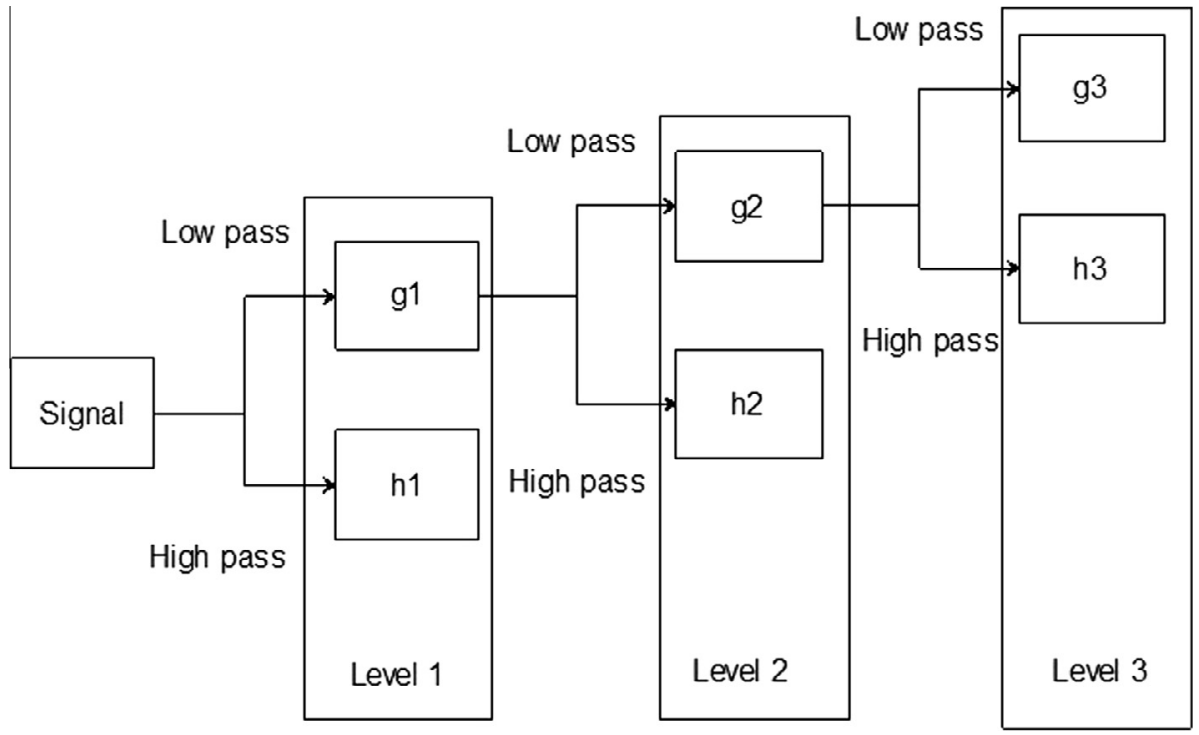

Fig. 6. A diagram of the steps performed within the wavelet filtering algorithm. 


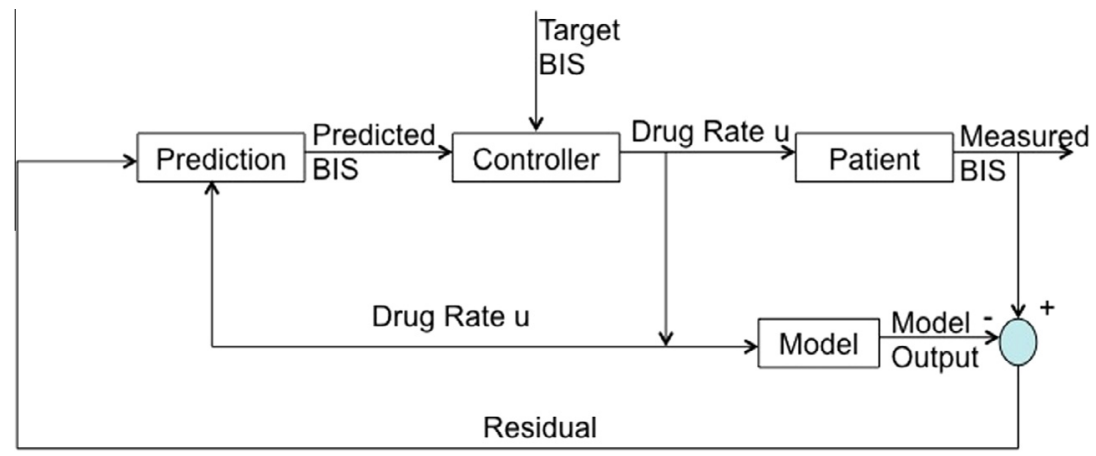

Fig. 7. MPC scheme for closed loop BIS regulation. Notice that this differs from the standard PID control scheme, since the MPC is an advanced control algorithm which requires the availability of a model for prediction, i.e. the controller receives two variables: target BIS and predicted BIS.

movement of the patient, etc. However, these effects have a stochastic character with non-zero average value, which can be modeled by a colored noise process:

$$
n(t)=\frac{C}{D} \cdot e(t),
$$

where $e(t)$ - uncorrelated (white) noise with zero mean value; $C$ and $D$ - monic polynomials of orders $n_{c}$ and $n_{d}$. Commonly, the disturbance filter (10) is defined as a pure integrator, to ensure zero steady state error. The relationship between $u(t)$ and $\hat{B I S}(t)$ is given by generic dynamic system models. In our case the input applied to the patient, $\left[\begin{array}{ll}u_{1} & u_{2}\end{array}\right]$, is a vector containing the Propofol and Remifentanil delivery rates. The prediction model is represented by (1) and (6). The process output is predicted at time instant $t$ over the prediction horizon $N_{2}$, based on the measurements available at that moment and the future outputs of the control signal, as schematically represented in Fig. 8. The values of the output predicted at the time instant $t$ over $k$ samples in the future are denoted by $(t+k \mid t)$ and defined as:

$$
B I S(t+k \mid t)=\hat{B I S}(t+k \mid t)+n(t+k \mid t) .
$$

Prediction of $B I S(t+k \mid t)$ and of $n(t+k \mid t)$ can be done respectively by recursion of the process model and by using filtering techniques on the noise model (10). The controller output is obtained by minimizing:

$$
J(U)=\sum_{k=N_{1}}^{N_{2}}\left[B I S^{*}(t+k \mid t)-\hat{B I S}(t+k \mid t)\right]^{2},
$$

where $B I S^{*}(t+k \mid t)$ is the desired reference trajectory, $B I S^{*}$ is the target BIS value and $N_{1}$ is the number of samples corresponding to the time delay (the prediction is calculated after the time delay). This cost function is a quadratic form in $U$, having the following structure:

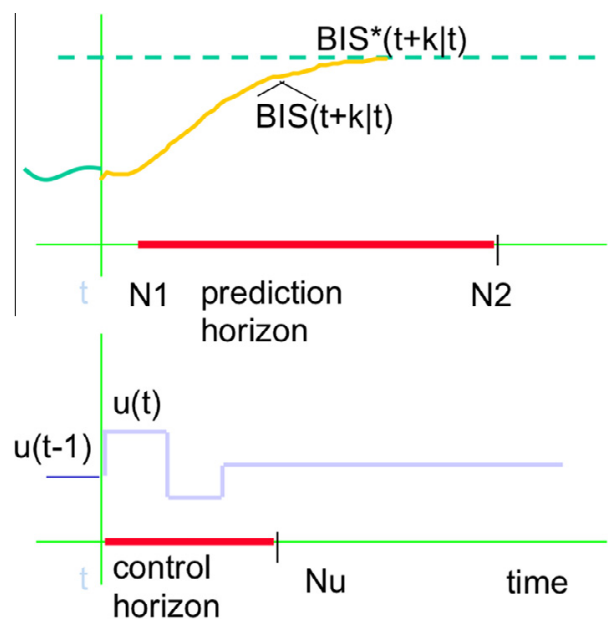

Fig. 8. A diagram of the concept of prediction in MPC; $u$ denotes the input, $y$ denotes the output. 


$$
J(U)=\left(\mathbf{B I S}^{*}-\overline{\mathbf{B I S}}-\mathbf{G} \cdot \mathbf{U}\right)^{T} \cdot\left(\mathbf{B I S} \mathbf{S}^{*}-\overline{\mathbf{B I S}}-\mathbf{G} \cdot \mathbf{U}\right),
$$

where $\overline{\mathbf{B I S}}$ is the predicted output of the response of the process model calculated if the postulated input would be kept constant at its value applied at time $(t-1)$. The postulated input is a choice that the user makes in the design of the controller and it corresponds to the simplest case, namely when the control horizon $N_{u}=1$ sample. Of course, the control horizon can take other values, but this only increases numerical complexity of the algorithm (i.e. matrix size) without significant performance improvement. For the specific case if control horizon of 1 sample, the $\mathbf{G}$ matrix is represented by:

$$
\mathbf{G}=\left[\begin{array}{ll}
m_{1} \cdot \operatorname{gProp}_{N_{1}} & m_{2} \cdot \operatorname{gRem}_{N_{1}} \\
\cdots & \cdots \\
m_{1} \cdot \operatorname{gProp}_{N_{2}} & m_{2} \cdot \operatorname{gRem}_{N_{2}}
\end{array}\right]
$$

where gProp $_{N_{1}} \ldots g$ Prop $_{N_{2}}$ and $g$ Rem $_{N_{1}} \ldots g$ gem $N_{2}$ are the coefficients of the unit step response of the PK-PD propofol model and PK-PD remifentanil model, respectively. Minimization w.r.t. $\mathbf{U}$ leads to the optimal solution:

$$
\mathbf{U}^{*}=\left[\mathbf{G}^{T} \cdot \mathbf{G}\right]^{-1} \cdot \mathbf{G}^{T} \cdot\left(\mathbf{B I S} \mathbf{S}^{*}-\overline{\mathbf{B I S}}\right)
$$

Only the first element in $\mathbf{U}^{*}$ is required in order to compute the actual control input applied to the process:

$$
u(t)=u_{\text {base }}(t \mid t)+\mathbf{U}^{*}(1)
$$

where the notation $(t \mid t)$ denotes the prediction made at time instant $t$ and given that $u_{\text {base }}$ are past predicted inputs to the process. At the next sampling instant $t+1$, the whole procedure is repeated taking into account the new measurement information $B I S(t+1)$.

The MPC-EPSAC strategy was implemented to control the level of BIS, using the model presented in Fig. 4 . Since we have two inputs and one output, when using the default cost function (12) we obtain an infinite number of solutions. The cost function can be extended in order to penalize the control movements and in this way the search region is restricted, leading to a unique solution:

$$
J(U)=\sum_{k=N_{1}}^{N_{2}}\left[B I S^{*}(t+k \mid t)-\hat{B I S}(t+k \mid t)\right]^{2}+\lambda_{1} \sum_{k=0}^{N_{2}-1}\left[U_{P r o p}(t+k \mid t)\right]^{2}+\lambda_{2} \sum_{k=0}^{N_{2}-1}\left[U_{R e m}(t+k \mid t)\right]^{2},
$$

where $\lambda_{1}$ and $\lambda_{2}$ are weighting parameters.

\section{Results}

\subsection{Filtering the real BIS signal}

The wavelet transform algorithm was used to recover the BIS signal from the noisy recorded data. The BIS signal was decomposed in different frequency bands, filtered and reconstructed, using the MatLab Wavelet toolbox. Five levels were adopted for decomposition, shown in Fig. 9, and applied on the BIS signals which vary in the time-frequency locus, as illustrated in Fig. 10 for one patient. The choice of the particular values in the decomposition levels is based on the dynamic response of the patient to drug infusion and to (surgical) stimuli. For instance, the threshold of $0.0031 \mathrm{~Hz}$ corresponds to a time interval of $300 \mathrm{~s}$. A typical surgical stimuli can increase BIS to dangerous values as fast as 30-130 s, thus the lowest decomposition level we have employed was of the order of $0.0015 \mathrm{~Hz}$.

\subsection{Identification from real data}

In order to identify the parameters online, an algorithm which selects the windows automatically was implemented. This algorithm has a better performance than the previous one since it takes into account the relation between the parameters $\left(\left|m_{1}\right|>\left|m_{2}\right|\right)$ and checks the values obtained during several iterations. Negative values were expected to be obtained for $m_{1}$ and $m_{2}$, since the relation between the effect site concentrations and BIS is inverse proportional.

The algorithm can be described as follows: The identification is initially performed on a window of 20 samples and then the window is increased with 10 samples. In case the identified parameters did not reach the upper limit of -0.1 (since both parameters should have negative values), the algorithm checks if the variation of the parameters is higher than a threshold of 0.5 (to avoid dithering) and if $\left|m_{1}\right|>\left|m_{2}\right|$. If these both conditions are fulfilled, the values of the parameters are recorded, and the whole procedure is repeated on a new window of 20 samples. In case the parameters reached the limits, the previous values of the parameters are used, and the algorithm re-iterated on the same window enlarged with 10 samples.

As an example, the result of the identification procedure applied on the data measured from patient \#1 is given in Fig. 11 . One may observe the variations in the estimated parameter values, suggesting that these parameters are indeed timevarying.

The validation against real data is presented in Fig. 12. When the first disturbance appears in patient's dynamics, after the first hour during their stay in the intensive care unit, the $U_{R e m}$ and $U_{\text {Prop }}$ rates start to increase. This suggest that the patient is 


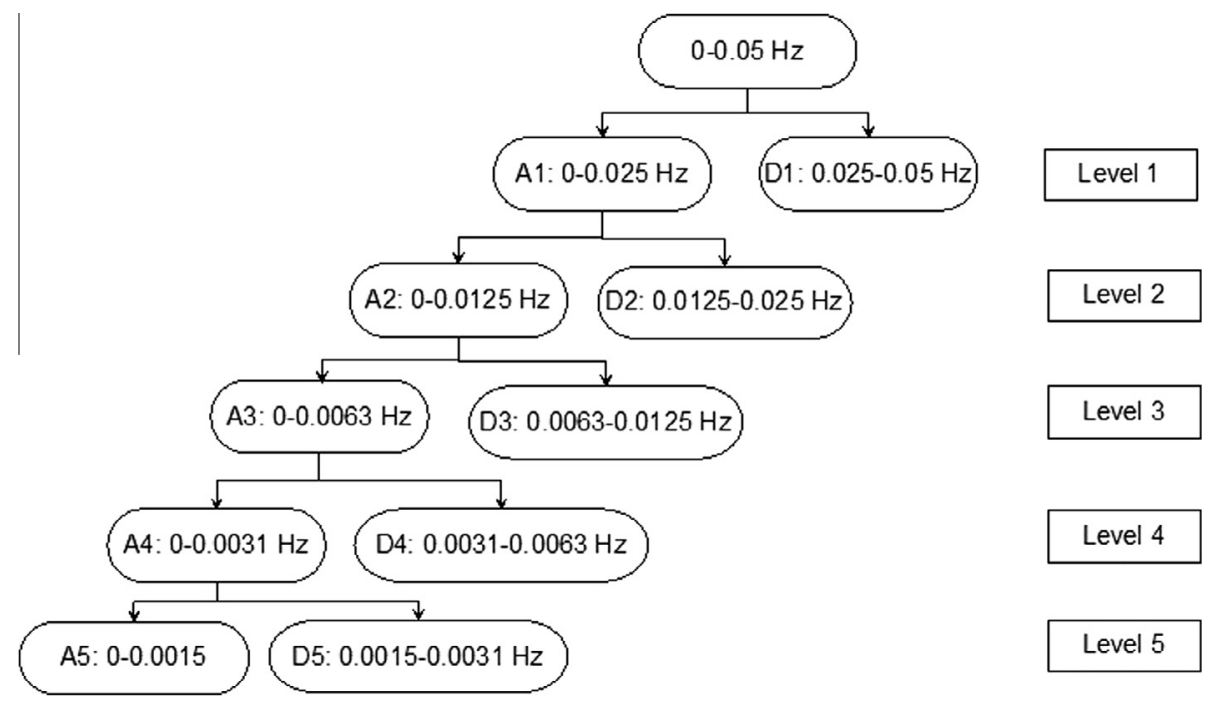

Fig. 9. Wavelet decomposition tree for filtering the noisy BIS signal.
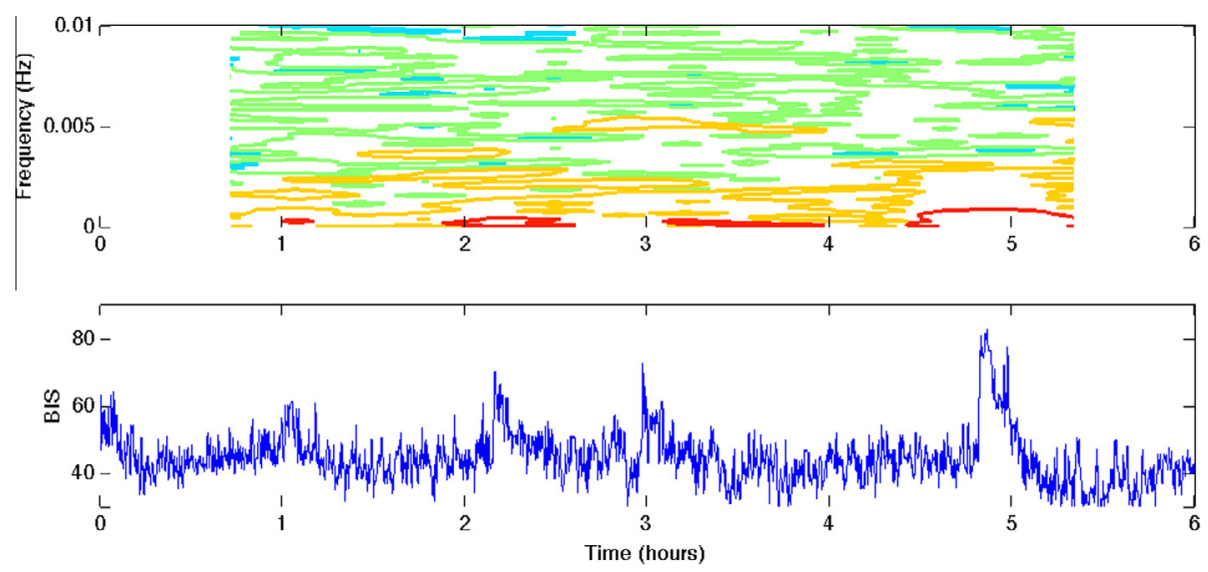

Fig. 10. An example of time-frequency analysis plot (top) and time signal of BIS (bottom) for one patient.
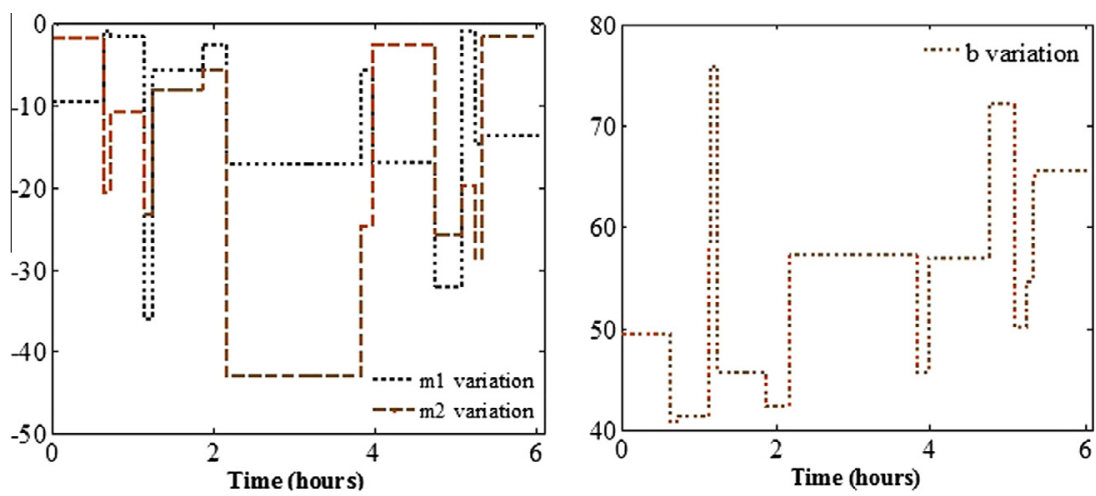

Fig. 11. Variation in the parameters estimated from data recorded from patient \#1.

less sensitive to the drug and needs higher infusion rates to maintain the same level of BIS values, visible in the higher values for $m_{1}$ and $m_{2}$. The control performance and the corresponding drug effect is successfully evaluated by the immediate 

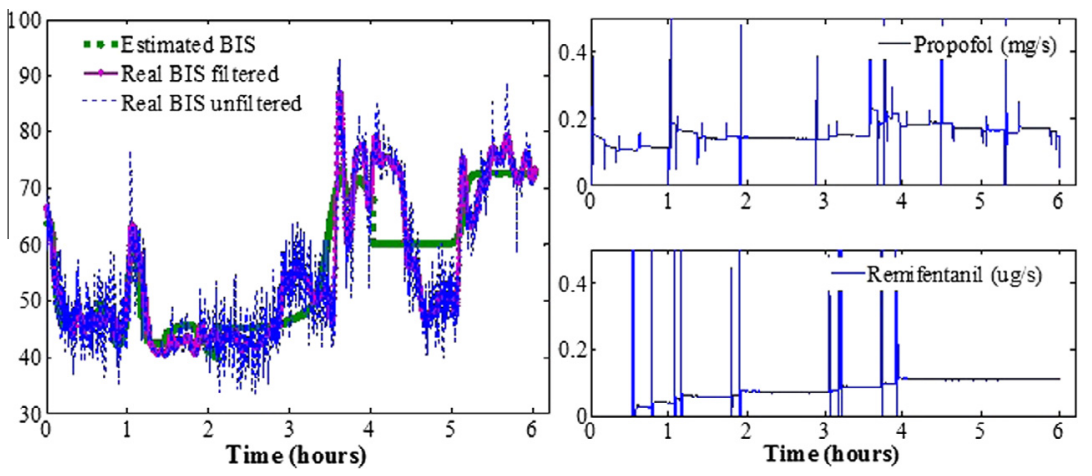

Fig. 12. Validation of identified model. Left: the real and estimated BIS signal. Right: the propofol and remifentanil records from patient \#1.

rejection of the disturbance by adding more drug into the patient and thus resulting in higher values of their blood concentrations.

The results of the identification procedure on the 5 patient records are listed in Table 2. When the patient does not receive any drug, the BIS is around $90-100 \%$. When they arrive in the intensive care unit, the patients are already sedated from the operation theater. This explains why the identified average value of $b$ is already around 50\%. Regarding the values obtained

Table 2

Averaged values from estimated model parameter values.

\begin{tabular}{llll}
\hline Patient & $m_{1}$ & $m_{2}$ & $b$ \\
\hline 1 & -18.12 & -13 & 54.74 \\
2 & -18.18 & -10.96 & 46.37 \\
3 & -12.12 & -10.82 & 36.12 \\
4 & -11.14 & -8.04 & 58 \\
5 & -12 & -3.7 & 63.87 \\
Nominal & $-14.31 \pm 3.52$ & $-9.30 \pm 3.59$ & $50.01 \pm 13.86$ \\
\hline
\end{tabular}
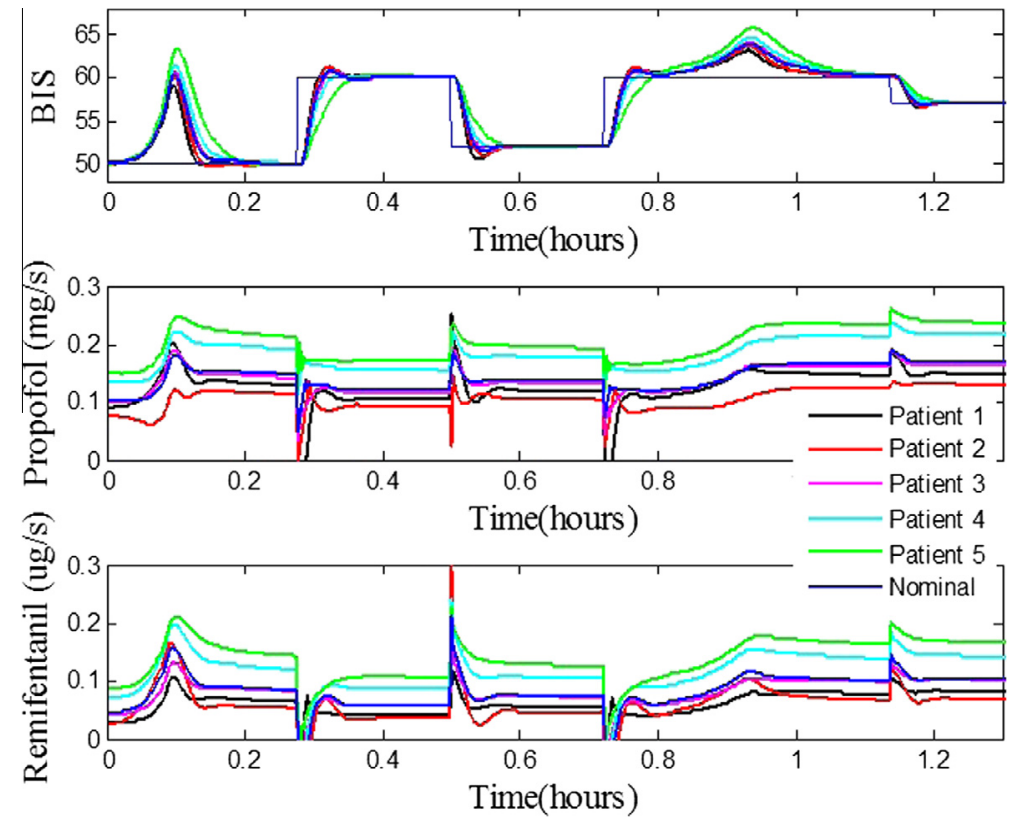

Fig. 13. Controller performance in closed loop under modeling errors and step disturbances. Modeling errors between the simulated patient model and the averaged patient model used for prediction are taken into account to prove robustness of the controller. Observe the different values of drug in steady state, showing that the control tackles well the inter-patient variability. 

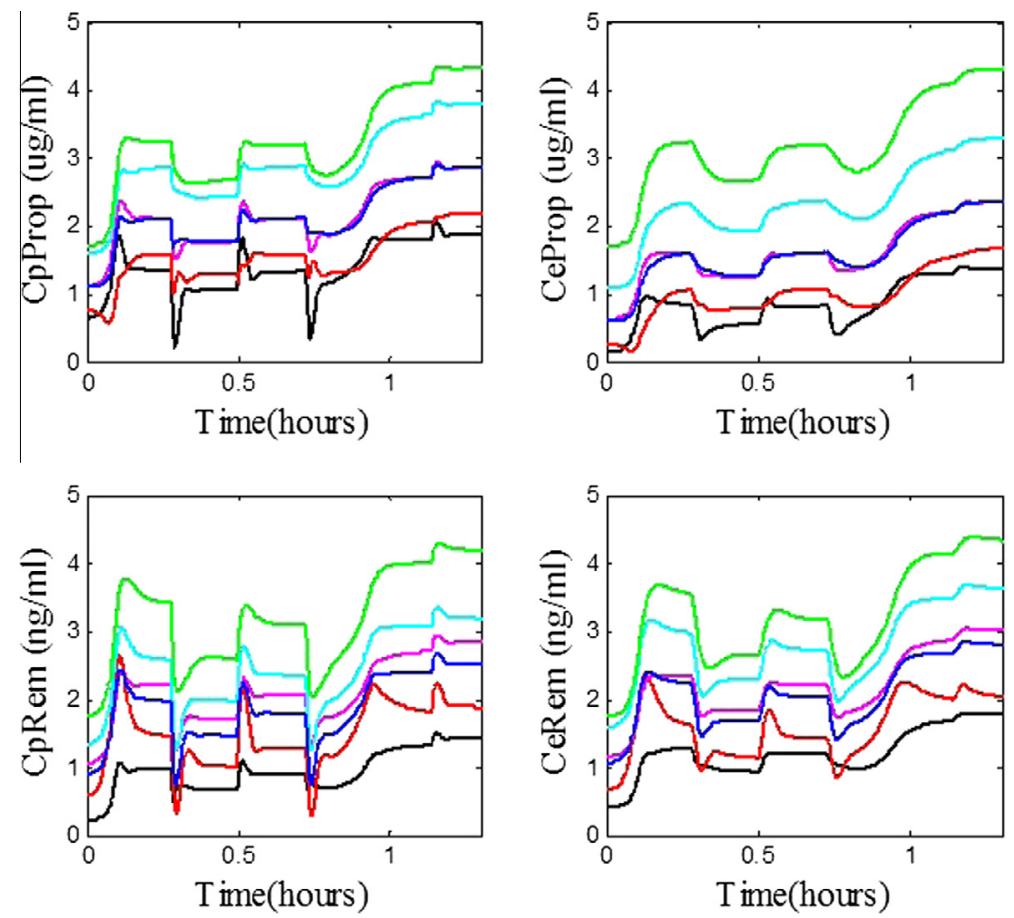

Fig. 14. Blood concentration $\left(C_{p}\right)$ and effect-site concentration $\left(C_{e}\right)$ obtained for different patients.

Table 3

Performance of the closed loop control.

\begin{tabular}{llll}
\hline Patient & Percentage undershoot & Settling time $(\mathrm{s})$ & Time elapsed to reject disturbance $(\mathrm{s})$ \\
\hline 1 & 2.73 & 120 & 531 \\
2 & 1.92 & 205 & 545 \\
3 & 0 & 144 & 640 \\
4 & 0 & 133 & 612 \\
5 & 0 & 216 & 702 \\
\hline
\end{tabular}

for $m_{1}$ and $m_{2}$, one can observe that patient \#5 has a higher sensitivity to propofol, compared with the others. Similarly, patients \#2 and \#3 have a higher sensitivity to remifentanil.

\subsection{Closed loop control: a simulation study}

The MPC-EPSAC algorithm was simulated using the prediction model from (1) and (6), with averaged values of Table 2. The optimal design of the controller was obtained for: $N_{1}=1, N_{u}=1, N_{2}=N_{1}+10$ samples, with a sampling period of $10 \mathrm{~s}$. The following minimal and maximal flow rates were imposed for the propofol and the remifentanil delivery pumps, respectively: $0-3.3 \mathrm{mg} / \mathrm{s}$ and $0-3.3 \mu \mathrm{g} / \mathrm{s}$. The weighting factors from the cost function in (17) were set to $\lambda_{1}=1000 ; \lambda_{2}=500$.

The closed loop control was simulated for different patients, in order to test the inter-patient variability and control performance for reference changes and disturbance rejection. For simulating each patient in a realistic manner, the corresponding parameters from Table 1 and Table 2 were employed. For prediction, the PK-PD parameters from Table 1 and the averaged values from Table 2 were employed. The disturbance rejection was evaluated for a step disturbance with the same amplitude as the one that appears in open-loop in case of patient \#1 after one hour. Two disturbances were introduced in the simulation, i.e. at $t=0.1$ and $t=0.8 \mathrm{~h}$. The results of the closed loop control simulations are presented in Fig. 13. It can be observed that the infusion rates are very close to the nominal ones depicted in Fig. 12.

The propofol and remifentanil concentrations estimated from the model for each patient are depicted in Fig. 14. These concentrations do not go beyond their safety limit of 4.5 of their respective units, as clinically imposed by the standardized patient safety protocol.

The controller performance for reference tracking and disturbance rejection was also analyzed. The percent of undershoot and the settling time when the reference is changing with an amplitude of $8 \%$ BIS are given in Table 3. The disturbance 
rejection time for a step disturbance with the amplitude $25 \%$ BIS is given as well. Both these values were chosen based on typical clinical practice.

From Figs. 13 and 14 we can conclude that patient \#5 has the lowest sensitivity to propofol and remifentanil. As a result, a high quantity of drug is delivered to the patient and a poor performance is obtained for disturbance rejection and reference tracking. For this patient significant modeling errors between the nominal model (used for prediction) and the identified model (used for simulating the real patient) occur: $10 \%$ error for $m_{1}$ and $65 \%$ for $m_{2}$. Indeed, the slope given by $m_{2}$ is more difficult to estimate due to the indirect effect from Remifentanil drug input to the BIS output value (recall here Fig. 5). Therefore, it is commendable that the controller has a good performance in closed loop despite such errors in prediction. In contrast, the fastest reference tracking is achieved in case of patient \#1 at the expense of a small undershoot and this is due to the high sensitivity to propofol and remifentanil.

\section{Limitations of this study}

A significant limitation of this study may be the low number of clinical data from which the model parameters were derived. However, the proposed model based predictive control strategy has an intrinsic robustness to model variations and stability has been shown to be guaranteed for significant gain variations in [4], i.e. variations between $1.6 * k-0.5 * k$, with $k$ the gain of the nominal patient model.

The results reported in this study suggest that the control performance has been shown to be more robust by $12 \%$ during induction phase as compared to the study in [4], where only Propofol was administered. The disturbance were not the same in both studies, so a performance comparison during maintenance is not possible.

\section{Conclusions}

The aim of this study was to present a pragmatic solution for modeling a two-drug synergic effect during general anesthesia. The drug interaction and their corresponding effect on the EEG signal was analysed by means of the standardized measure of depth of sedation, i.e. the bispectral index. A simplified linear multiple-input single-output model for patient's response is proposed. Two PK-PD models are used in clinical practice and their parameters are a priori known from biometric details of the patient: age, weight, gender and height. An interaction model was proposed and, based on the clinical data, the parameters of this linear model were identified by means of time-frequency filtering algorithms. The identified averaged model proved to suffice for hypnosis level prediction when propofol and remifentanil are administered, with estimated values very close to those measured in clinical practice. This approach was further supported by the simulation of the closed loop model based predictive control algorithm, with drug infusion rates and drug concentrations close to clinical practice.

\section{Acknowledgment}

This work has been funded by IWT TBM project number 060776 supported by the Flemish Institute for Innovation through Science and Technology during 2007-2009. The authors acknowledge ir. Tom De Smet (Demed, Temse, Belgium) for his support with the RUGLOOP II software platform and Mr. Patrick De Baets, RN, for his help during the clinical trials.

\section{References}

[1] Absalom A, De Keyser R, Struys MMRF. Closed loop anesthesia: are we getting close to finding the Holy Grail? Anesth Analg 2011;112(3):516-8 [editorial Special Issue].

[2] De Keyser R, Ionescu CM. A no-nonsense control engineering approach to anesthesia control during induction phase. In: Proceedings of IFAC Int Conf on Biology and Medicine, Special Session on Model Formulation, decision support and control of depth of anesthesia: towards a multivariable paradigm, Budapest, Hungary; 2012. p. 379-384. doi: http://dx.doi.org/10.3182/20120829-3-HU-2029.00077. [29-31 August 2012].

[3] Ritchie RG, Ernst EA, Pate BL, Pearson JD, Shepherd LC. Closed-loop control of an anesthesia delivery system: development and animal testing. IEEE Trans Biomed Eng 1987;34(6):437-43.

[4] Ionescu C, De Keyser R, Torrico BC, De Smet T, Struys M, Normey-Rico JE. Robust predictive control strategy applied for propofol dosing using BIS as a controlled variable during anesthesia. IEEE Trans Biomed Eng 2008;55:2161-70.

[5] De Smet T, Struys MM, Neckebroek MM, Van den Hauwe K, Bonte S, Mortier EP. The accuracy and clinical feasibility of a new bayesian-based closedloop control system for propofol administration using the bispectral index as a controlled variable. Anesth Analg 2008;107:1200-10.

[6] Struys MM, De Smet T, Versichelen LF, Van De Velde S, Van den Broecke R, Mortier EP. Comparison of closed-loop controlled administration of propofol using Bispectral Index as the controlled variable versus standard practice controlled administration. Anesthesiology 2001;95:6-17.

[7] Yeragania VK, Radhakrishnab RKA, Ramakrishnanb KR, Srinivasanb SH. Measures of LLE of heart rate in different frequency bands: a possible measure of relative vagal and sympathetic activity. Nonlinear Anal Real World Appl 2004;5(3):441462.

[8] Roya PK, Chatterjeea AN, Greenhalghb D, Khanc QJA. Long term dynamics in a mathematical model of HIV-1 infection with delay in different variants of the basic drug therapy model. Nonlinear Anal Real World Appl 2013;14(3):16211633.

[9] Nguyen-Ky TT, Wen Peng, Li Y. Measuring and reflecting depth of anesthesia using wavelet and power spectral density. IEEE Trans Inf Technol Biomed 2011;15(4):630-9.

[10] Struys M, De Smet T, Versichelen L. System and method for adaptive drug delivery. US Patent 6605072. 2003.

[11] Schnider TW, Minto CF, Gambus PL, Andresen C, Goodale DB, Youngs EJ. The influence of method of administration and covariates on the pharmacokinetics of Propofol in adult volunteers. Anesthesiology 1998;88:11701182.

[12] Schnider TW, Minto CF, Shafer SL, Gambus PL, Andresen C, Goodale DB, Youngs EJ. The influence of age on Propofol pharmacodynamics. Anesthesiology 1999;90:1502-16. 
[13] Minto CF, White M, Morton N, Kenny GN. Pharmacokinetics and pharmacodynamics of remifentanil. II model application,. Anesthesiology 1997;86:24-33.

[14] Minto CF, Schnider TW, Egan TD, Youngs E, Lemmens HJ, Gambus PL, Billard V, Hoke JF, Moore KH, Hermann DJ, Muir KT, Mandema JW, Shafer SL. Influence of age and gender on the pharmacokinetics and pharmacodynamics of remifentanil. I. model development. Anesthesiology 1997;86:10-23.

[15] Minto CF, Schnider TW, Short TG, Gregg KM, Gentilini A, Shafer SL. Response surface model for anesthetic drug interactions. Anesthesiology 2000;92:1603-16.

[16] Ionescu C, De Keyser R, Hodrea R, Sendoya D, Robayo F. Variable time delay estimation for anesthesia control during intensive care. IEEE Trans Biomed Eng 2011;58(2):363-9.

[17] Burrus CS, editor. Efficient Fourier Transform and Convolution Algorithms, Advanced Topics in Signal Processing. Englewood Cliffs, NJ: Prentice-Hall; 1998. p. 199245.

[18] Mallat S. A theory for multiresolution signal decomposition: the wavelet representation. IEEE Trans Pattern Anal Mach Intell 1989;11(7):674-93.

[19] Yelneedi S, Samavedham L, Rangaiah GP. Advanced control strategies for the regulation of hypnosis with propofol. Ind Eng Chem Res 2009;48:3880-97.

[20] De Keyser RMC. Model Based Predictive Control for Linear Systems. UNESCO Encyclopaedia of Life Support Systems Article contribution 6.43.16.1. Eolss Publishers Co Ltd, Oxford. ISBN 0. 2003.

[21] Kaul HL, Bharti N. Monitoring depth of anaesthesia. Indian J Anaesth 2002:46(4):323-32.

[22] Struys M, Vereecke H, Moerman A, Jensen EW, Verhaeghen D, De Neve N, et al. Ability of the bispectral index, autoregressive modelling with exogenous input-derived auditory evoked potentials, and predicted Propofol concentrations to measure patient responsiveness during anesthesia with Propofol and Remifentanil. Anesthesiology 2003;99:802-12. 